FGI-99-3

math.QA/9903093

\title{
Two Dimensional Fractional Supersymmetry from the Quantum Poincaré Group at Roots of Unity
}

\author{
Hadji AHMEDOV ${ }^{a, \text { f }}$ and Ömer F. DAYI ${ }^{a, b, \not 2}$ \\ a) Feza Gürsey Institute, \\ P.O.Box 6, 81220 Çengelköy-Istanbul, Turkey. \\ b)Physics Department, Faculty of Science and Letters, Istanbul Technical University, \\ 80626 Maslak-Istanbul, Turkey.
}

\begin{abstract}
A group theoretical understanding of the two dimensional fractional supersymmetry is given in terms of the quantum Poincaré group at roots of unity. The fractional supersymmetry algebra and the quantum group dual to it are presented and the pseudounitary, irreducible representations of them are obtained. The matrix elements of these representations are explicitly constructed.
\end{abstract}

\footnotetext{
${ }^{1}$ E-mail address: hagi@gursey.gov.tr

${ }^{2}$ E-mail addresses: dayi@gursey.gov.tr and dayi@itu.edu.tr.
} 


\section{Introduction}

Quantum algebras at roots of unity were seen to be useful in formulations of some physical systems whose theoretical understanding is not very clear [1]. Obviously, to achieve a complete understanding of the role of these algebras in applications to physical systems one should know the quantum groups which are dual to them. Indeed, in [2] $E_{q}(1,1)$ at roots of unity and in [3] $S L_{q}(2, \mathbb{R})$ at roots of unity were constructed. In the formulation of these groups one is obliged to introduce some new variables which are the generalized Grassmannians $\eta_{ \pm}$satisfying $\eta_{ \pm}^{p}=0$ where $p$ is a positive integer. On the other hand these coordinates were used to obtain superspace realizations of the fractional supersymmetry charges [4]-[7]. Although some algebraic properties of the two dimensional fractional supersymmetry were discussed in [5], the correct behaviour under the Lorentz generator could be obtained in terms of some restrictions and a spectral parameter.

A group theoretical understanding of the fractional supersymmetry appears to be lacking. Our aim is to shed some light on the group theoretical aspects of the fractional supersymmetry in two dimensions. Hence, guided by the formulation of the two dimensional quantum Poincaré algebra at roots

of unity [2], we introduce the fractional supersymmetry algebra $U_{F}$ endowed with a Hopf algebra structure. We present the quantum group $\mathcal{A}_{F}$ which is its dual. Pseudo-unitary, irreducible corepresentations of $\mathcal{A}_{F}$ are given and the matrix elements of them are explicitly calculated. We, then, define the pseudo-unitary quasi-regular corepresentation of $\mathcal{A}_{F}$ and the corresponding *-representation of $U_{F}$.

\section{Fractional supersymmetry algebra and its dual}

Let us deal with the two dimensional Poincaré algebra $U(e(1,1))$ generated by $P_{ \pm}$and $H$ possessing the commutators

$$
\left[P_{+}, P_{-}\right]=0,\left[P_{ \pm}, H\right]= \pm i P_{ \pm},
$$

and the involutions

$$
P_{ \pm}^{*}=P_{ \pm}, H^{*}=H .
$$

The two dimensional fractional supersymmetry generators $p_{ \pm}$are defined to satisfy

$$
p_{ \pm}^{p}=P_{ \pm}
$$


where $p$ is a positive integer, without any condition on commutation relation of $p_{+}$with $p_{-}$. Obviously, the simplest choice is $p_{+} p_{-}=p_{-} p_{+}$. Thus, the quantum Poincaré algebra at roots of unity $U_{q}(e(1,1))$ with $q^{p}=1, p$ is an odd, positive integer, generated by $p_{ \pm}$and $\kappa$ satisfying

$$
\left[p_{+}, p_{-}\right]=0, \kappa p_{ \pm}=q^{ \pm 1} p_{ \pm} \kappa, \kappa^{p}=1_{U},
$$

with the involutions

$$
p_{ \pm}^{*}=p_{ \pm}, \kappa^{*}=\kappa
$$

suits well with our purposes. $1_{U}$ denotes the unit element of the algebra.

The two dimensional fractional supersymmetry algebra denoted $U_{F} \equiv$ $\left(U(e(1,1)), U_{q}(e(1,1))\right)$ is generated by $P_{ \pm}, H, p_{ \pm}, \kappa$ satisfying (11)-(5) and moreover, the commutation relations

$$
[\kappa, H]=0,\left[p_{ \pm}, H\right]= \pm \frac{i}{p} p_{ \pm}
$$

The latter is the consequence of (11) and (3).

The basis elements of $U_{F}$ are

$$
\phi^{n m k r s l} \equiv p_{+}^{n} p_{-}^{m} \kappa^{k} P_{+}^{r} P_{-}^{s} H^{l},
$$

where $n, m, k, r, s, l$ are positive integers.

We can equip $U_{F}$ with the Hopf algebra structure

$$
\begin{array}{lll}
\Delta\left(P_{ \pm}\right)=P_{ \pm} \otimes 1_{U}+1_{U} \otimes P_{ \pm}, & \varepsilon\left(P_{ \pm}\right)=0, & S\left(P_{ \pm}\right)=-P_{ \pm}, \\
\Delta(H)=H \otimes 1_{U}+1_{U} \otimes H, & \varepsilon(H)=0, & S(H)=-H, \\
\Delta\left(p_{ \pm}\right)=p_{ \pm} \otimes \kappa+\kappa^{-1} \otimes p_{ \pm}, & \varepsilon\left(p_{ \pm}\right)=0, & S\left(p_{ \pm}\right)=-q^{ \pm 1} p_{ \pm}, \\
\Delta(\kappa)=\kappa \otimes \kappa, & \varepsilon\left(\kappa^{ \pm 1}\right)=1, & S\left(\kappa^{ \pm 1}\right)=\kappa^{\mp 1} .
\end{array}
$$

Now, we would like to present the groups which are dual to the algebras considered above.

The $*$-algebra $\mathcal{A}(E(1,1))$ of infinitely differentiable functions on the two dimensional Poincaré group $E(1,1)$ is dual to the algebra $U(e(1,1))$. For any $f\left(z_{+}, z_{-}, \lambda\right) \in \mathcal{A}(E(1,1))$ we have the involution

$$
z_{ \pm}^{*}=z_{ \pm}, \lambda^{*}=\lambda
$$

where

$$
\left(\begin{array}{ccc}
e^{\lambda} & 0 & z_{+} \\
0 & e^{-\lambda} & z_{-} \\
0 & 0 & 1
\end{array}\right) \in E(1,1)
$$


The two dimensional quantum Poincaré group at roots of unity [2] is the *-algebra $\mathcal{A}\left(E_{q}(1,1)\right)$ with $q^{p}=1$, generated by $\eta_{ \pm}, \delta$ satisfying

$$
\begin{array}{r}
\eta_{-} \eta_{+}=q^{2} \eta_{+} \eta_{-}, \quad \eta_{ \pm} \delta=q^{2} \delta \eta_{ \pm} \\
\eta_{ \pm}^{p}=0, \quad \delta^{p}=1_{A} \\
\eta_{ \pm}^{*}=\eta_{ \pm}, \quad \delta^{*}=\delta
\end{array}
$$

where $1_{A}$ is the unit element of $\mathcal{A}$. The dual of $U_{F}$ is the $*$-algebra $\mathcal{A}_{F}=$ $\mathcal{A}(E(1,1)) \times \mathcal{A}\left(E_{q}(1,1)\right)$ with the Hopf algebra structure

$$
\begin{aligned}
& \Delta\left(\eta_{ \pm}\right)=\eta_{ \pm} \otimes 1_{A}+\delta^{ \pm 1} e^{ \pm \lambda / p} \otimes \eta_{ \pm}, \quad \varepsilon\left(\eta_{ \pm}\right)=0, \quad S\left(\eta_{ \pm}\right)=-\delta^{\mp 1} \eta_{ \pm}, \\
& \Delta(\delta)=\delta \otimes \delta \\
& \varepsilon\left(\delta^{ \pm 1}\right)=1, \quad S\left(\delta^{ \pm 1}\right)=\delta^{\mp 1}, \\
& \Delta(\lambda)=\lambda \otimes 1_{A}+1_{A} \otimes \lambda, \\
& \varepsilon(\lambda)=-\lambda, \quad S(\lambda)=-\lambda, \\
& \Delta\left(z_{ \pm}\right)=z_{ \pm} \otimes 1_{A}+e^{ \pm \lambda} 1_{A} \otimes z_{ \pm}+(-1)^{\frac{p+1}{2}} \sum_{n=1}^{p-1} \frac{q^{ \pm n^{2}}}{[p-n] ![n] !} \eta_{ \pm}^{p-n} \delta^{ \pm n} e^{ \pm \lambda n / p} \otimes \eta_{ \pm}^{n}, \\
& S\left(z_{ \pm}\right)=-z_{ \pm}, \quad \varepsilon\left(z_{ \pm}\right)=0 .
\end{aligned}
$$

We use the symmetric $q-$ number

$$
[n]=\frac{q^{n}-q^{-n}}{q-q^{-1}}
$$

and the $q$-factorial $[n] !=[n][n-1] \cdots[1]$.

Since any function of $\mathcal{A}(E(1,1))$ can locally be expanded in Taylor series, there is a local basis of $\mathcal{A}_{F}$ given by

$$
a^{n m k t s l} \equiv \eta_{+}^{n} \eta_{-}^{m} \zeta(k, \delta) z_{+}^{t} z_{-}^{s} \lambda^{l}
$$

where $n, m, k, t, s, l$ are positive integers and we defined

$$
\zeta(m, \delta) \equiv \frac{1}{p} \sum_{n=0}^{p-1} q^{-n m} \delta^{n} .
$$

The duality relations between $\mathcal{A}_{F}$ and $U_{F}$ are

$\left\langle\phi^{n m k t s l}, a^{n^{\prime} m^{\prime} k^{\prime} t^{\prime} s^{\prime} l^{\prime}}\right\rangle=i^{n+m+t+s+l} q^{\frac{n-m}{2}-n m} l ! t ! s ![n] ![m] ! \delta_{n n^{\prime}} \delta_{m m^{\prime}} \delta_{t t^{\prime}} \delta_{s s^{\prime}} \delta_{l l^{\prime}} \delta_{k+n+m, k^{\prime}}$. 


\section{Pseudo-unitary, irreducible corepresentations of $\mathcal{A}_{F}$}

Let $C_{0}^{\infty}(\mathbb{R})$ be the space of all infinitely differential functions of finite support in $\mathbb{R}$ and $P(t)$ denote the algebra of polynomials in $t$ subject to the conditions $t^{p}=1$ and $t^{*}=t$. The linear map

$$
\pi_{r}\left(U_{F}\right): C_{0}^{\infty}(\mathbb{R}) \times P(t) \rightarrow C_{0}^{\infty}(\mathbb{R}) \times P(t)
$$

given as

$$
\begin{aligned}
\pi_{r}\left(p_{ \pm}\right) f(x) a(t) & =(-r)^{1 / p} e^{ \pm x / p} t^{ \pm 1} f(x) a(t), \\
\pi_{r}\left(P_{ \pm}\right) f(x) a(t) & =-r e^{ \pm x} f(x) a(t), \\
\pi_{r}(H) f(x) a(t) & =-i \frac{d}{d x} f(x) a(t), \\
\pi_{r}(\kappa) f(x) a(t) & =f(x) a(q t),
\end{aligned}
$$

defines the irreducible representation of $U_{F}$ in $C_{0}^{\infty}(\mathbb{R}) \times P(t)$.

Let us introduce the following Hermitian forms for the space $C_{0}^{\infty}(\mathbb{R}) \times$ $P(t)$

$$
\begin{aligned}
& \left(f_{1}, f_{2}\right)=\int_{-\infty}^{+\infty} d x f_{1}(x) \overline{f_{2}(x)} \\
& \left(a_{1}, a_{2}\right)=\Phi\left(a_{1}(t) a_{2}^{*}(t)\right)
\end{aligned}
$$

where

$$
\Phi\left(t^{s}\right)=\delta_{s, 0(\bmod \mathrm{p})} .
$$

$C_{0}^{\infty}(\mathbb{R})$ endowed with the norm induced by (16) leads to the Hilbert space of the square integrable functions on $\mathbb{R}$. On the other hand $P(t)$ with the pseudo-norm $\|a\|^{2} \equiv(a, a)$ is the pseudo-Euclidean space with $\frac{p+1}{2}$ positive and $\frac{p-1}{2}$ negative signatures[2]. Now, one can verify that $\pi_{r}$ defines pseudounitary, irreducible $*$-representation of $U_{F}$ for real $r$.

By making use of the duality relations (14), we can derive from (15) the irreducible corepresentations of $\mathcal{A}_{F}$ as

$$
T_{r}(f(x) a(t))=\sum_{n, m, k=0}^{p-1} \sum_{t, s, l=0}^{\infty} \frac{a^{n m k t s l} \pi_{r}\left(\phi^{n m k t s l}\right) f(x) a(t)}{\left\langle\phi^{n m k t s l}, a^{n m k t s l}\right\rangle},
$$

which is pseudo-unitary for real $r$. 
Consider the Fourier transform of $f(x) \in C_{0}^{\infty}(\mathbb{R})$

$$
F(\nu)=\int_{-\infty}^{+\infty} f(x) e^{\nu x} d x
$$

This integral converges for any complex $\nu . F(\nu)$ is an analytic function and moreover, satisfies $\left(\nu=\nu_{1}+i \nu_{2}\right)$

$$
\left|F\left(\nu_{1}+i \nu_{2}\right)\right|<K e^{c\left|\nu_{1}\right|},
$$

for some real constants $K$ and $c$. Then we can write the inverse transform as

$$
f(x)=\frac{1}{2 \pi i} \int_{c-i \infty}^{c+i \infty} F(\nu) e^{-\nu x} d \nu .
$$

The Fourier transform of $T_{r}(19)$ yields the pseudo-unitary corepresentation in the space of functions $F(\nu) a(t)$ as

$$
Q_{r}\left(F(\nu) t^{k}\right)=\int_{c-i \infty}^{c+i \infty} d \mu \sum_{l=0}^{p-1} Q_{k l}^{r}(\nu, \mu, g) F(\mu) t^{l}
$$

where we denoted the variables as $g \equiv\left(g_{0} ; g_{p}\right)=\left(z_{+}, z_{-}, \lambda ; \eta_{+}, \eta_{-}, \delta\right)$ and the kernel $Q_{k l}^{r}$ is

$$
\begin{aligned}
\text { for } l \geq k, & \\
Q_{k l}^{r}(\nu, \mu, g)= & \left(q^{-1 / 2} \eta_{+}\right)^{l-k} \omega_{l-k}^{r}(\xi) \delta^{k} K_{l-k}^{r}\left(\nu, \mu, g_{0}\right) \\
& +\omega_{p+k-l}^{r}(\xi)\left(q^{1 / 2} \eta_{-}\right)^{p+k-l} \delta^{k} K_{l-k-p}^{r}\left(\nu, \mu, g_{0}\right) \\
\text { for } l<k, \quad & \\
Q_{k l}^{r}(\nu, \mu, g)= & \left(q^{-1 / 2} \eta_{+}\right)^{p+l-k} \omega_{p+l-k}^{r}(\xi) \delta^{k} K_{p+l-k}^{r}\left(\nu, \mu, g_{0}\right) \\
& +\omega_{k-l}^{r}(\xi)\left(q^{1 / 2} \eta_{-}\right)^{k-l} \delta^{k} K_{l-k}^{r}\left(\nu, \mu, g_{0}\right) .
\end{aligned}
$$

We introduced, in terms of $\xi=q \eta_{+} \eta_{-}$, the polynomials

$$
\omega_{s}^{r}(\xi)=\sum_{m=0}^{p-s-1} \frac{\left(i r^{1 / p}\right)^{2 m+s}}{[n] ![m+s] !}\left(q^{s} \xi\right)^{m}
$$

and the functions $K_{s}^{r}$ are

$$
K_{s}^{r}\left(\nu, \mu, g_{0}\right)=\frac{1}{2 \pi i} e^{\mu \lambda} \int_{-\infty}^{+\infty} e^{i r\left(e^{x} z_{+}+e^{-x} z_{-}\right)+x(\nu-\mu+s / p)} d x
$$


By utilizing the analog of polar coordinates $\rho>0, \beta \in \mathbb{R}$, the pseudoEuclidean plane defined by the axis $z_{-}=0$ and $z_{+}=0$ can be studied in terms of the quadrants

$$
\begin{aligned}
& \text { Quad.1: } z_{+} z_{-}>0, z_{ \pm}=\frac{1}{2} \rho e^{ \pm \beta}, \quad \text { Quad.2: } z_{+} z_{-}<0, z_{ \pm}= \pm \frac{1}{2} \rho e^{ \pm \beta}, \\
& \text { Quad.3: } z_{+} z_{-}>0, z_{ \pm}=\frac{-1}{2} \rho e^{ \pm \beta}, \quad \text { Quad.4: } z_{+} z_{-}<0, z_{ \pm}=\mp \frac{1}{2} \rho e^{ \pm \beta} .
\end{aligned}
$$

In these quadrants (27) will lead to the Hankel functions $H_{\nu}^{(1)}, H_{\nu}^{(2)}$ or cylindrical functions of imaginary argument $K_{\nu}$ :

$$
\begin{array}{ll}
\text { Quad.1: } & K_{s}^{r}\left(\nu, \mu, g_{0}\right)=\frac{1}{2} e^{(\mu-\nu-s / p)\left(\beta+\frac{\pi i}{2}\right)+\mu \lambda} H_{\mu-\nu-s / p}^{(1)}(r \rho), \\
\text { Quad.2: } & K_{s}^{r}\left(\nu, \mu, g_{0}\right)=\frac{1}{2} e^{(\mu-\nu-s / p)\left(\beta-\frac{\pi i}{2}\right)+\mu \lambda} H_{\mu-\nu-s / p}^{(2)}(r \rho), \\
\text { Quad.3: } & K_{s}^{r}\left(\nu, \mu, g_{0}\right)=\frac{1}{\pi i} e^{(\mu-\nu-s / p)\left(\beta+\frac{\pi i}{2}\right)+\mu \lambda} K_{\mu-\nu-s / p}(r \rho), \\
\text { Quad.4: } & K_{s}^{r}\left(\nu, \mu, g_{0}\right)=\frac{1}{\pi i} e^{(\mu-\nu-s / p)\left(\beta-\frac{\pi i}{2}\right)+\mu \lambda} K_{\mu-\nu-s / p}(r \rho),
\end{array}
$$

with the condition $-1<\operatorname{Re}(\nu-\mu+s / p)<1$. [8].

\section{Quasi-regular corepresentation of $\mathcal{A}_{F}$ and *-representation of the fractional supersymmetry algebra}

The comultiplication

$$
\Delta: \mathcal{A} \rightarrow \mathcal{A}_{F} \otimes \mathcal{A}
$$

defines the pseudo-unitary left quasi-regular corepresentation of $\mathcal{A}_{F}$ in its subspace $\mathcal{A}$ consisting of the finite sums

$$
X=\sum_{s} a_{s}\left(\eta_{+}, \eta_{-}\right) f_{s}\left(z_{+}, z_{-}\right)
$$

where $a_{s}\left(\eta_{+}, \eta_{-}\right)$are polynomials in $\eta_{+}, \eta_{-}$and $f_{s}\left(z_{+}, z_{-}\right) \in C_{0}^{\infty}\left(\mathbb{R}^{2}\right)$. The space $\mathcal{A}$ can be endowed with the hermitian form

$$
(X, Y)=\mathcal{I}_{E}\left(X Y^{*}\right)
$$

$X, Y \in \mathcal{A}$ and the linear functional $\mathcal{I}_{E}: \mathcal{A} \rightarrow \mathbb{C}$

$$
\mathcal{I}_{E}(X)=\sum_{s} \mathcal{I}\left(a_{s}\right) \mathcal{I}_{C}\left(f_{s}\right)
$$


is the left invariant integral where 2]

$$
\begin{aligned}
\mathcal{I}\left(\eta_{+}^{n} \eta_{-}^{m}\right) & =q^{-1} \delta_{n, p-1} \delta_{m, p-1} \\
\mathcal{I}_{C}\left(f_{s}\right) & =\int_{-\infty}^{+\infty} d z_{+} d z_{-} f_{s}\left(z_{+}, z_{-}\right)
\end{aligned}
$$

The right representation of the fractional supersymmetry algebra $U_{F}$ corresponding to the quasi-regular representation (28),

$$
\mathcal{R}(\phi) X=(\phi \otimes i d) \Delta(X)
$$

$\phi \in U_{F}$, is a $*-$ representation

$$
(\mathcal{R}(\phi) X, Y)_{E}=\left(X, \mathcal{R}\left(\phi^{*}\right) Y\right)_{E}
$$

because the hermitian form (29) is defined in terms of the left invariant integral (30).

The right representations on the variables $\eta_{ \pm}$and $f\left(z_{+}, z_{-}\right)$can explicitly be written as

$$
\begin{array}{ll}
\mathcal{R}\left(p_{ \pm}\right) \eta_{ \pm}^{k}=i q^{ \pm \frac{1}{2}}[k] \eta_{ \pm}^{k-1}, & \mathcal{R}\left(p_{ \pm}\right) \eta_{\mp}^{k}=0 \\
\mathcal{R}(\kappa) \eta_{ \pm}^{k}=q^{ \pm k} \eta_{ \pm}^{k}, & \mathcal{R}(H) \eta_{ \pm}^{n}= \pm \frac{i n}{p} \eta_{ \pm}^{n} \\
\mathcal{R}\left(p_{ \pm}\right) f=i q^{ \pm \frac{1}{2}} \frac{(-1)^{\frac{p+1}{2}}}{[p-1] !} \eta_{ \pm}^{p-1} \frac{d f}{d z_{ \pm}}, & \mathcal{R}\left(P_{ \pm}\right) f=i \frac{d f}{d z_{ \pm}} \\
\mathcal{R}(\kappa) f=f, & \mathcal{R}(H) f=i z_{+} \frac{d f}{d z_{+}}-i z_{-} \frac{d f}{d z_{-}}
\end{array}
$$

In terms of the following relations satisfied by the right representation $\mathcal{R}$

$$
\begin{aligned}
\mathcal{R}\left(\phi \phi^{\prime}\right) & =\mathcal{R}\left(\phi^{\prime}\right) \mathcal{R}(\phi), \\
\mathcal{R}\left(p_{ \pm}\right)(X Y) & =\mathcal{R}\left(p_{ \pm}\right) X \mathcal{R}(\kappa) Y+\mathcal{R}\left(\kappa^{-1}\right) X \mathcal{R}\left(p_{ \pm}\right) Y, \\
\mathcal{R}(\kappa)(X Y) & =\mathcal{R}(\kappa) X \mathcal{R}(\kappa) Y \\
\mathcal{R}(H)(X Y) & =\mathcal{R}(H) X Y+X \mathcal{R}(H) Y
\end{aligned}
$$

we can define the action of an arbitrary operator $\mathcal{R}(\phi)$ on any function in $\mathcal{A}$.

The quantum algebra which we deal with possesses one Casimir element $C=p_{+} p_{-}$. As the complete set of commuting operators we can choose $\mathcal{R}(C)$, $\mathcal{R}(H), \mathcal{R}(\kappa)$ and $\mathcal{L}(H), \mathcal{L}(\kappa)$ where $\mathcal{L}(\phi)$ is the left representation of the element $\phi$ defined similar to (33) with the interchange of $\phi$ with the identity $i d$. One can easily observe that $\mathcal{L}(H) X=0$ and $\mathcal{L}(\kappa) X=X$ for any $X \in \mathcal{A}$, 
so that, in the space $\mathcal{A}$ the matrix elements can be labeled as $D_{n \nu, m \mu}^{r}$. Indeed, in terms of the kernel $Q_{m n}^{r}$ (24) one observes that

$$
D_{n \nu, 00}=Q_{0 n}^{r}(\nu, 0, g),
$$

$n \in[0, p-1]$, satisfy

$$
\begin{aligned}
\mathcal{R}(\kappa) D_{n \nu, 00} & =q^{n} D_{n \nu, 00} \\
\mathcal{R}(H) D_{n \nu, 00} & =-i(\nu+n / p) D_{n \nu, 00} \\
\mathcal{R}(C) D_{n \nu, 00} & =c^{2} D_{n \nu, 00} \\
\mathcal{R}\left(p_{+}\right) D_{n \nu, 00} & =c D_{n+1 \nu, 00} \\
\mathcal{R}\left(p_{-}\right) D_{n \nu, 00} & =c D_{n-1 \nu, 00}, \\
\mathcal{R}\left(P_{ \pm}\right) D_{k \nu, 00} & =-r D_{k \nu \pm 1,00},
\end{aligned}
$$

where $c=r^{1 / p} q$ and we introduced the notation $D_{p \nu, 00} \equiv D_{0 \nu+1,00}$ and $D_{-1 \nu, 00} \equiv D_{p-1 \nu-1,00}$.

The right representation obtained in (34) can be used to write the supercharge operators $\mathcal{R}\left(p_{ \pm}\right)$in the superspace given by $\eta_{+}, z_{+}$or $\eta_{-}, z_{-}$as

$$
\mathcal{R}\left(p_{ \pm}\right)=i q^{ \pm \frac{1}{2}} D_{ \pm}^{q}+\frac{(-1)^{\frac{p+1}{2}}}{[p-1] !} \eta_{ \pm}^{p-1} \frac{d}{d z_{ \pm}},
$$

where $D_{ \pm}^{q}$ are q-derivatives with respect to $\eta_{ \pm}$. This is the same with the realization of supercharges given in [4]-[7], obtained in terms of $q$-calculus. 


\section{References}

[1] Ö.F. Dayi, J. Phys. A: Math. Gen, 31 (1998) 3523 and the references therein.

[2] H. Ahmedov, Analysis on the 2-Dim Quantum Poincaré Group at Roots of Unity, math.QA/9808110.

[3] H. Ahmedov and Ö.F. Dayi, J. Phys. A 32 (1999) 1531.

[4] S. Durand, Mod. Phys. Lett A8 (1993) 2323.

[5] E.H. Saidi, M.B. Sedra and J. Zerouaoui, Class. Quantum Grav. 12 (1995) 1567.

[6] N. Mohammedi, Mod. Phys. Lett A 10 (1995) 1287.

[7] R.S. Dunne, A.J. Macfarlane, J.A. de Azcárraga and J.C. Pèrez Bueno, Int. J. Mod. Phys. A12, (1997). 3275.

[8] N.Ja. Vilenkin and A.U. Klimyk, Representation of Lie groups and special functions. Vols.1-3, (Dordrecht:Kluwer) 1992. 